\title{
Infrared Fixed Point of SU(3) gauge theory with 12 flavor staggered fermions
}

\section{Kenji Ogawa*t}

National Chiao-Tung University

E-mail: bqawaknjegmail.com

\begin{abstract}
We present our study to identify the location of the fixed point of SU(3) gauge theory with 12 flavors. We show a method to extract the renormalization group beta function from the data of lattice renormalized coupling using a simultaneous fit with respect to bare coupling and lattice size. We show some examples of application of this method to available data from step scaling study, and compare the location of infrared fixed point.
\end{abstract}

The 30 International Symposium on Lattice Field Theory - Lattice 2012,

June 24-29, 2012

Cairns, Australia

\footnotetext{
* Speaker.

${ }^{\dagger}$ moved to Chung Yaun Christian University
} 


\section{Introduction}

Technicolor model is a model in which extra gauge fields and fermion fields couples to the Standard Model particles. It gives an alternative mechanism to give the mass of standard model particles without elementary Higgs particles. To be consistent with the results of experiment, small enough flavor changing neutral current and large enough standard model fermion mass, this model need a property called "Walking Technicolor", that the running of technicolor coupling constant becomes very slow at the intermediate region while keeping asymptotic freedom and spontaneous breaking at the ultraviolet region and the infrared region. The main issue is that such a theory really exists or not and, if it does, the details of the theory, e.g., the representation, the number of colors and fermion flavors of the theory. To clarify this issue, all possible models need to be studied one by one non-perturbatively, especially the theory which is considered to locate near the edge of conformal window, the region of the parameter space such that the theory have infrared fixed point. To identify the location of the edge of the conformal window, non-perturbative study of lattice simulation is a useful tool and a lot of work have been done on this issue.[(])

When one study the running of coupling constant with lattice simulation, the step scaling function are widely used. In this article, we reconsider the analysis method of step scaling function. Especially, I propose an analysis method which does not specify the size of step scaling.

This paper is organized as follows. At first, we review the step scaling function. Next, we introduce the new analysis method. Then, we show the application of the method using the available data. In the end, we give the summary and comments.

\section{Step Scaling Function}

The step scaling function gives the growth of the coupling constant with respect to the reference scale. Provided that a scheme to define the renormalized coupling constant are given and the coupling constant is $u$ at a reference momentum scale $1 / L,{ }^{1}$ the step scaling function $\sigma(u, s)$ gives the coupling constant at the scale $1 /(s L)$.

$$
\sigma(u ; s)=g^{2}(1 /(s L)) \text { with } u=g^{2}(1 / L) .
$$

The step size $s$ is fixed in the step scaling function given by the practical lattice study (typically $s \sim 2$ ). Given the step scaling function with fixed $s$, to see the coupling constant in the wide range of scale, one can iteratively apply the step scaling function, say,

$$
u_{i+1}=\sigma\left(u_{i}, s\right), \text { with } u_{i} \stackrel{\text { def }}{=} u\left(1 /\left(s^{i} L\right)\right) .
$$

The relation between renormalization group beta function $\beta(u)=L \frac{d u}{d L}$ and step scaling function is

$$
\int_{u}^{\sigma(u, s)} \frac{d u}{\beta(u)}=-\log s
$$

when $\log s \ll 1$, beta function are approximated as

$$
\frac{\sigma(u, s)-u}{\log s} \sim \beta(u)
$$

\footnotetext{
${ }^{1}$ In this article, $u$ is always the squared of renormalized coupling $u=g^{2}$.
} 
To take the continuum limit of the step scaling function from the lattice simulation, one uses following method. (1). Set a scheme such that the reference scale is given by the linear extent of the space time. (2). Prepare the lattice with lattice sites $\hat{L}_{0}$ with a set of bare parameter $\hat{\mathbf{g}}$ and calculate renormalized coupling $u_{\text {lattice }}$ at that system. Then prepare the lattice with lattice sites $s \hat{L}_{0}$ with a same set of bare parameter $\hat{\mathbf{g}}$ and calculate renormalized coupling. It gives the lattice step scaling function $\Sigma\left(u, s, \hat{L}_{0}\right)$.

$$
\Sigma\left(u, s, \hat{L}_{0}\right)=g_{\text {lattice }}^{2}\left(\hat{\mathbf{g}}^{(\mathbf{0})}, s \hat{L}_{0}\right) \text { with } u=g_{\text {lattice }}^{2}\left(\hat{\mathbf{g}}^{(\mathbf{0})}, \hat{L}_{0}\right)
$$

The difference of $\Sigma(u, s, \hat{L})$ and $\sigma(u, s)$ are the lattice artifact which vanish at $\hat{L} \rightarrow \infty$ limit. The continuum limit is taken by calculating $\Sigma\left(u, s, \hat{L}_{i}\right)$ using different set of lattice $\left(\hat{L}_{i}, s \hat{L}_{i}\right)$ while keeping the same renormalized coupling $u$ for $\hat{L}_{i}$ lattice by tuning the set of bare parameter $\hat{\mathbf{g}}^{(i)}$. The bare parameter are always set to be the same for $\hat{L}_{i}$ lattice and $s \hat{L}_{i}$ lattice.

$$
\sigma(u, s)=\lim _{\hat{L}_{i} \rightarrow \infty} \Sigma\left(u, s, \hat{L}_{i}\right)=\lim _{\hat{L}_{i} \rightarrow \infty} g_{\text {lattice }}^{2}\left(\hat{\mathbf{g}}^{(i)}, s \hat{L}_{i}\right) \text { with } u=g_{\text {lattice }}^{2}\left(\hat{\mathbf{g}}^{(i)}, \hat{L}_{i}\right)
$$

\section{Method of Analysis}

Before describing the method to extract the beta function, we gave the proof of the consistency equation (B.5) of multi-step lattice step scaling function. We restrict ourself to use only massless fermions with chirally-symmetric lattice formulation ( staggered or overlap fermions ). Then the tunable parameter is bare coupling $g_{0}$ only. By definition,

$$
\Sigma\left(g_{\text {lattice }}^{2}\left(g_{0}^{2}, \hat{L}\right) ; s, \hat{L}\right)=g_{\text {lattice }}^{2}\left(g_{0}^{2}, s \hat{L}\right) .
$$

By substituting $\left(s=s_{1}, \hat{L}=\hat{L}_{0}\right),\left(s=s_{2}, \hat{L}=s_{1} \hat{L}_{0}\right)$ and $\left(s=s_{1} s_{2}, \hat{L}=\hat{L}_{0}\right)$ into this equation, one get three equations.

$$
\begin{gathered}
\Sigma\left(g_{\text {lattice }}^{2}\left(g_{0}^{2}, \hat{L}_{0}\right) ; s_{1}, \hat{L}_{0}\right)=g_{\text {lattice }}^{2}\left(g_{0}^{2}, s_{1} \hat{L}_{0}\right) \\
\Sigma\left(g_{\text {lattice }}^{2}\left(g_{0}^{2}, s_{1} \hat{L}_{0}\right) ; s_{2}, s_{1} \hat{L}_{0}\right)=g_{\text {lattice }}^{2}\left(g_{0}^{2}, s_{2} s_{1} \hat{L}_{0}\right) \\
\Sigma\left(g_{\text {lattice }}^{2}\left(g_{0}^{2}, \hat{L}_{0}\right) ; s_{2} s_{1}, \hat{L}_{0}\right)=g_{\text {lattice }}^{2}\left(g_{0}^{2}, s_{2} s_{1} \hat{L}_{0}\right)
\end{gathered}
$$

By comparing these equations, one get the consistency condition as,

$$
\Sigma\left(u ; s_{1} s_{2}, \hat{L}\right)=\Sigma\left(\Sigma\left(u ; s_{1}, \hat{L}\right) ; s_{2}, s_{1} \hat{L}\right),
$$

with $u=g_{\text {lattice }}^{2}\left(g_{0}^{2}, \hat{L}\right)$.

Now, I describe the analysis method. At first, parameterize the beta function using coefficients $c_{l}$ as $\beta(u)=\beta\left(u ; c_{l}\right)$. By solving the integral equation (2.3]), one get the expression of the step scaling function with $c_{l}$ as $\sigma\left(u, s ; c_{l}\right)$. Next, we obtain the lattice step scaling function by adding lattice artifact term with parameters $k_{j}$ as $f\left(u, s, k_{j}\right)$ which vanish at $\hat{L} \rightarrow \infty$. In practice, we neglect higher order of $1 / \hat{L}$ by assuming that the simulation is done near enough the continuum limit. In our analysis, the form of the lattice artifact $f\left(u, s, k_{j}\right)$ are highly constrained by requiring Eq. 
(B.5) to be satisfied. Besides the parameterization of $\Sigma$ with $c_{l}$ and $k_{j}$, in this analysis one need to parameterize one set of the renormalized coupling constant which is given by the same lattice site $L_{0}$ (parameterized with $h_{m}$ ).

$$
g_{\text {lattice }}^{2}\left(g_{0}^{2}, \hat{L}_{0}\right)=G\left(g_{0}^{2} ; h_{m}\right)
$$

In this work, we use the quadratic polynomial $g_{\text {lattice }}^{2}\left(g_{0}^{2}, \hat{L}_{0}\right)=h_{0}+h_{1} g_{0}^{2}+h_{2} g_{0}^{4}$. Then, with the lattice step scaling function $\Sigma(u, s, \hat{L})$, one can fit $g_{\text {latt }}$ as,

$$
g_{\text {lattice }}^{2}\left(g_{0}^{2}, \hat{L}\right)=\Sigma\left(g_{\text {lattice }}^{2}\left(g_{0}^{2}, \hat{L}_{0}\right) ; s=\hat{L} / \hat{L}_{0}, \hat{L}_{0}\right)
$$

By this fitting, we get the parameters $c_{l}, k_{j}$ and $h_{m}$, then we can reconstruct the beta function using $c_{l}$. We emphasize that this fitting are done only for limited region of $g_{0}$ or $u$. To get the information of the beta function in the wide range, we divide the region into small pieces and get the set of $c_{l}$ for each region. In this way, we can make a "patch" of the beta function.

Now, we show a simplest example, beta function do not depend on $u$ and parameterized by one constant $\beta(u)=c$. Then the step scaling function is given by

$$
\sigma(u ; s)=u+c \log s
$$

Now, for simplicity, we only add lattice artifact term proportional to $(1 / \hat{L})$. (Adding higher order terms is trivial.) The lattice step scaling function is

$$
\Sigma(u ; s, \hat{L})=u+c \log s+\frac{1}{\hat{L}} f(u, s)
$$

$f(u, s)$ is the coefficient of lattice artifact. Here, we assume that we can neglect $u$ dependence of $f(u, s)$. Then using the consistency condition [3.5, we obtain the form of $f(u, s)=f(s)$ as,

$$
f(s)=k\left(\frac{1}{s}-1\right)
$$

This is the only one expression to satisfy [3.5], when $u$ dependence are omitted and only $(1 / L)$ term are considered.

One can do the similar calculation for the linear beta function, $\beta(u)=c\left(u-u_{0}\right)$. The lattice step scaling function is written as,

$$
\Sigma(u ; s, \hat{L})=u_{0}+\left|u-u_{0}\right| s^{c}+\sum_{j=1}^{j_{\mathrm{MAX}}} \frac{k_{j}}{(\hat{L})^{j}}\left(\frac{1}{s^{j}}-s^{c}\right)
$$

Here, one can choose the order of lattice artifact by changing $j_{\text {MAX }}$. Now, it will be helpful to summarize the assumption which have made in the analysis.

1.) Fit of the beta function $\beta(u)$ is done in a limited region of $u$.

2.) Lattice artifact term is a analytic function of $u$ and $s$.

3.) Lattice artifact term is up to a few order of $1 / \hat{L}$

4.) For the lattice artifact term $f(u, s, \hat{L}), u$ dependence are omitted.

5.) $g^{2}\left(L_{0}\right)$ is described as polynomial of $g_{0}$ in a small region. 

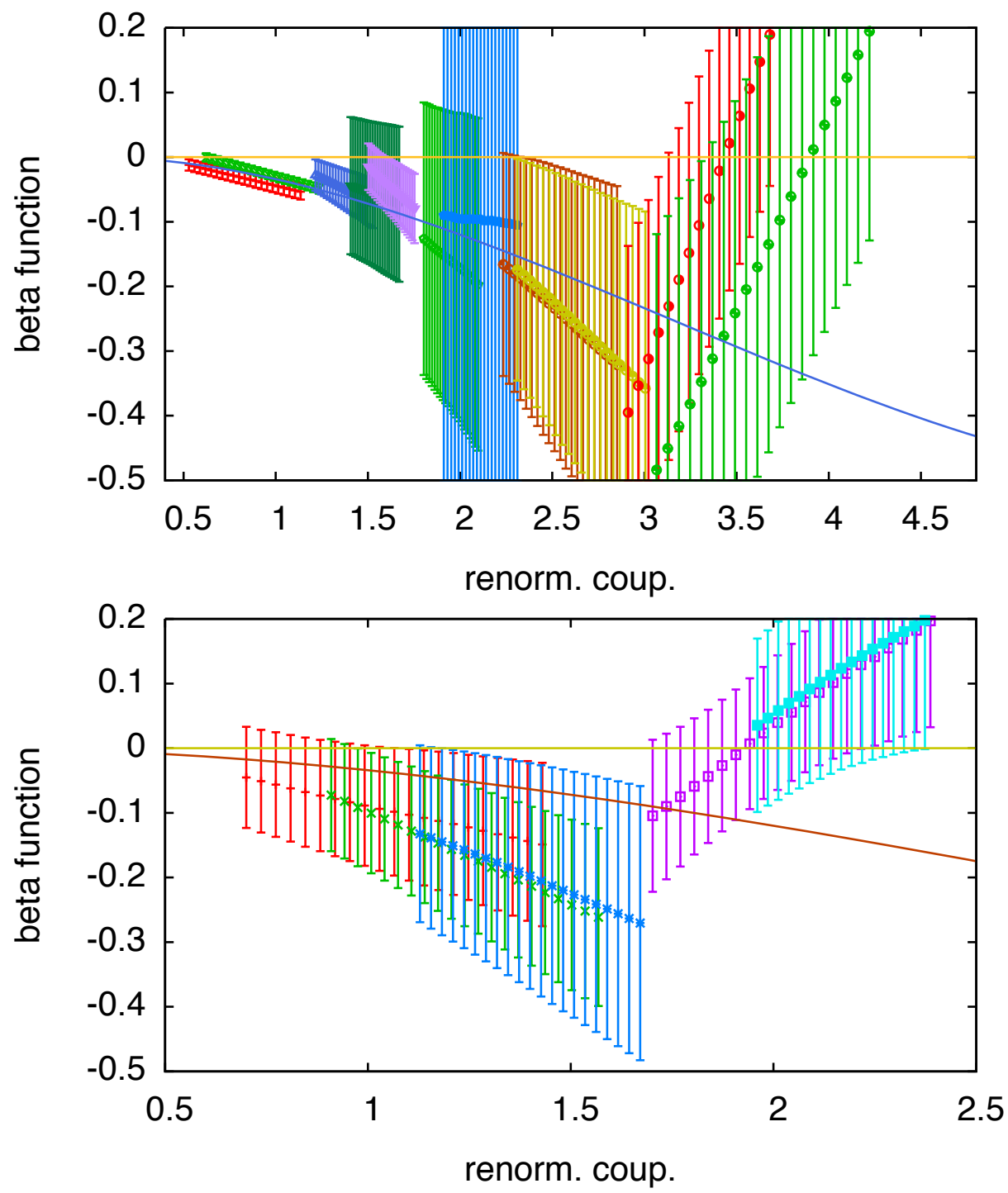

Figure 1: The reconstructed beta function from the data in [2] (top) and [3] (bottom). The band with different color shows the different patch, they are reconstructed using different set of the parameter $c_{l}(c$ and $u_{0}$ for this case).

\section{Application}

Here, we show the application of the method. We analyzed the data of TABLE III in [2] and TABLE IV in [B]. In both case massless staggered fermion are used. We used linear beta function B.T and we include the lattice artifact up to second order. The result of reconstructed beta function is shown in Fig $\mathbb{W}$. For both case, we see the beta function consistent to be zero in the infrared region as mentioned in the original articles.

\section{Summary and Comments}

We provided an alternative method of the analysis of the running of the renormalized coupling 
constant. The basis of our analysis are four Equations In the application, our method gives the consistent results of original article with conventional step scaling function analysis. In the analysis method here, some assumption we put will not be justified in general (ex. Linear beta function, omitting $u$ dependence of lattice artifact term). To overcome these, the further development the analysis method keeping the same approach is going on.

\section{Acknowledgments}

We acknowledge the author of [ [ $]$ ] and [B] for showing the useful data on the articles. We acknowledge George Fleming, C-J. David Lin, Takeshi Yamazaki, Hiroshi Ohki, Masafumi Kurachi, and Yasumichi Aoki for discussion, comments and advice.

\section{References}

[1] Ethan T. Neil, Exploring Models for New Physics on the Lattice, PoS LATTICE2011 (2011), 009.

[2] Thomas Appelquist, George T. Fleming, and Ethan T. Neil, Lattice Study of Conformal Behavior in SU(3) Yang-Mills Theories, Phys.Rev. D79 (2009), 076010.

[3] C.-J. David Lin, Kenji Ogawa, Hiroshi Ohki, and Eigo Shintani, Lattice study of infrared behaviour in SU(3) gauge theory with twelve massless flavours, JHEP 1208 (2012), 096. 